\title{
EL AGUA REgENERADA COMO FUENTE ALTERNATIVA SOSTENIBLE DE RIEGO EN AGRICULTURA EN EUROPA: EL CASO DE ANDALUCÍA
}

\author{
Julio Berbel $^{\mathrm{a},}$ Rafael Casielles$^{\mathrm{b}}$, Alfonso Expósito $^{\mathrm{c}}$, Antonia Lorenzo $^{\mathrm{a}, \mathrm{b}}$ y Enrique Mesa-Pérez ${ }^{\mathrm{a} *}$ \\ ${ }^{a}$ Universidad de Córdoba, Córdoba, emesa@uco.es ${ }^{b}$ Bioazul, ${ }^{c}$ Universidad de Málaga
}

\begin{abstract}
Resumen
En la presente comunicación se resumen los resultados obtenidos en el proyecto SuWaNu-Europe al respecto de las debilidades, amenazas, fortalezas y oportunidades de la regeneración de aguas en Europa y más concretamente en la región de Andalucía. A través de dos encuestas se identifican los principales componentes del DAFO en las ocho regiones analizadas por el proyecto y la relevancia o impacto de éstas sobre la potencial reutilización de agua en agricultura. Los resultados nos destacan que la percepción social, el coste y el marco regulatorio son los principales elementos que abordar si se pretende fomentar el uso del agua regenerada en agricultura. Sin embargo, estos elementos no afectan por igual a todas las regiones. Se pueden reunir las regiones en dos clústeres: el primero formado por Alemania, Bélgica, Bulgaria, Grecia y Portugal, cuyas regiones se han caracterizado por tener una mayor disponibilidad de agua. Las principales preocupaciones de los agentes claves han sido el alto coste de implementar la reutilización, la falta de infraestructuras y la gestión de los riesgos. El segundo clúster, formado por España, Francia e Italia, con mayor concienciación debido a la escasez previa, ha destacado la disponibilidad de infraestructuras y la oportunidad que supone disponer de una fuente de riego alternativa de forma constante todo el año. En cualquier caso, ambos clústeres destacan la necesidad de concienciar a la sociedad para que la reutilización tenga éxito.
\end{abstract}

\section{Palabras claves}

Reutilización, regadío, sostenibilidad, agricultura, Andalucía

\section{Introducción}

La frecuencia e intensidad de las sequías es cada vez mayor, provocando que la escasez de agua esté dejando de ser un problema exclusivo de los países del sur y se esté convirtiendo en un problema global (Hristov, Barreiro-Hurle, Salputra, Blanco, y Witzke, 2021). La instalación de sistemas de riego más eficientes o el fomento de fuentes alternativas como la desalación o la reutilización son alternativas que permiten disponer de los recursos hídricos de una forma más eficiente ayudando a paliar los efectos del cambio climático (Berbel y Esteban, 2019; Morote, Olcina, y Hernández, 2019).

La Unión Europea lleva varios años promoviendo iniciativas que ayuden a reducir el consumo de agua o permitan el acceso a nuevas fuentes alternativas, ejemplo de ello son la Directiva Marco de Agua (European Commission, 2000) o recientemente el Green Deal (European Commission, 2019). En esta comunicación nos centraremos en la reutilización como fuente alternativa de agua. Recientemente, el Parlamento Europeo y el Consejo de la Unión Europea han aprobado el Reglamento EU 2020/741 de requisitos de mínimos para la reutilización del agua (European Parliament, 2020). El objetivo del Reglamento es fomentar el uso del agua regenerada para uso agrícola. Estudios previos demostraban que a pesar de la idoneidad del agua regenerada para paliar la escasez (Shannon et al., 2008), la falta de apoyo social podía provocar el fracaso de proyectos de reutilización, como ocurrió en California y Australia (Mainali et al., 2011). En España contamos con una tradición de reutilización amplia, por ejemplo el Plan Nacional de Reutilización de aguas (MARM, 2010) ya establecía como meta para 2021 la reutilización del 35\% de las aguas tratadas, sin embargo, aún estamos lejos, ya que apenas hemos alcanzado el 10\% nacional o el 5.93\% de Andalucía (SuWaNu Europe Project, 2019a). Debemos añadir que la depuración de aguas residuales aún es deficiente provocando que la Unión Europea haya multado a España recientemente por este motivo.

Sin embargo, la aprobación del Reglamento europeo ha provocado que el Gobierno de España se encuentre redactando el Plan DSEAR para la reutilización de aguas o la Junta de Andalucía su Pacto andaluz por el agua, con especial atención a la reutilización como fuente alternativa de agua. Todas estas iniciativas legislativas ponen el foco sobre la reutilización de agua, permitiendo el desarrollo de las estrategias a corto y medio plazo. Para apoyar las diferentes iniciativas nacionales, surge SuWaNu-Europe Project (2019), una red temática financiada por el programa europeo Horizonte H2020, que tiene por objetivo la promoción de nuevas estrategias que fomenten el uso de agua regenerada en agricultura. El proyecto se centra en crear una red con los principales actores de cada una de las regiones incluidas en el proyecto: Andalucía (España), Braunshweig (Alemania), Tesalónica (Grecia), Plovdiv (Bulgaria), Alentejo (Portugal), Cuenca del río Po (Italia), Occitania (Francia), Amberes y Limburgo (Bélgica). A estas ocho regiones se suman equipos de 
Chipre e Israel como proyectos de referencia en la regeneración de agua, que asesorarán y colaborarán como uno más en el proyecto.

La presente comunicación pretende compartir el trabajo llevado a cabo en SuWaNu-Europe (SuWaNu Europe Project, 2019a, 2020) resumiendo los resultados del trabajo de algunos investigadores al respecto de las debilidades, amenazas, fortalezas y oportunidades de la reutilización de agua en Andalucía (MesaPérez, Expósito, Casielles, y Berbel, 2020) y del análisis clúster de las regiones europeas analizadas siguiendo los resultados del análisis DAFO (Mesa-Pérez y Berbel, 2020). Por ello se presenta un resumen del DAFO a nivel europeo y los resultados principales encontrados, para después profundizar sobre los resultados del análisis en Andalucía.

\section{Metodología}

El análisis DAFO realizado en el proyecto permitió conocer las principales barreras y oportunidades a las que se enfrentaba la reutilización en las regiones europeas analizadas (SuWaNu Europe Project, 2020). La metodología empleada fue la realización de una encuesta entre los agentes claves identificados en la primera fase del proyecto para cada una de las regiones (SuWaNu Europe Project, 2019a). Aunque el número de entrevistados varió en cada región, fueron entrevistados más de 60 agentes claves entre todas las regiones. La metodología constó de dos pasos, el primero de ellos, una encuesta sobre cuáles eran las principales debilidades, amenazas, fortalezas y oportunidades de la reutilización de agua en la región en cuestión. Este trabajo fue revisado por los integrantes del proyecto SuWaNu-Europe. El segundo paso fue una encuesta, formato Likert 1-5 para valorar la relevancia de cada elemento identificado, mostrando poca relevancia (1) o mucha relevancia (5). Además, la escala Likert permitía incluir un valor neutro (3) para aquellos agentes que tuvieran dudas sobre su respuesta (Barua, 2013; Croasmun y Ostrom, 2011). Finalmente, una vez obtenidos los resultados, se realizó un análisis clúster para conocer si los países se encontraban agrupados en función de los resultados del DAFO.

\section{Resultados}

El resumen de los resultados principales de los trabajos se centrará por un lado en destacar el resultado del análisis DAFO a nivel europeo y la clusterización de las regiones en función de las similitudes o diferencias de las percepciones. Por otro lado, se analizará en mayor profundidad las características de Andalucía como región de referencia de nuestro país en SuWaNu-Europe.

En función de estos resultados encontramos que las barreras se enmarcan en tres grupos principalmente: la percepción social, el coste y el marco regulatorio. La primera de las barreras está relacionada con la percepción social de que el riego de los cultivos con agua regenerada puede suponer un riesgo para la salud pública, tanto consumidores como comercializadores y distribuidores se muestran reticentes a consumir y comercializar los productos regados con agua regenerada. Sin embargo, los estándares de calidad del agua tratada por el terciario incluidos en el reglamento europeo supondrá que el agua regenerada tenga mayor calidad que la de muchos ríos españoles. La segunda de las barreras, el coste del agua regenerada provoca que muchos agricultores prefieran seguir usando aguas superficiales o subterráneas. Los planteamientos a los que ha tenido acceso el proyecto manifiestan como el uso que se pretende dar del agua regenerada es de fuente sustitutiva de los recursos convencionales, de forma que se permita la recuperación, o al menos reducción, de los acuíferos sobreexplotados. Sin embargo, los agricultores se oponen en parte, al preferir el agua regenerada como fuente alternativa en casos de escasez o necesidad y no fuente de uso constante. Finalmente, los encuestados convinieron que el marco regulatorio actual impide agilizar el proceso de concesiones de agua regenerada y tampoco establecía claros estándares de la calidad exigida al agua.

$\mathrm{El}$ análisis de clúster nos permite reunir a las diferentes regiones en dos grupos. Por un lado, el clúster formado por Alemania, Bélgica, Bulgaria, Grecia y Portugal y, por otro lado, el formado por España, Francia e Italia. La diferencia principal entre ambos grupos es la disponibilidad de agua. El primer clúster incluye regiones que tradicionalmente han dispuesto de agua y se caracteriza por su preocupación por la gestión de riesgos, el coste energético o la falta de infraestructuras. El segundo clúster, formado por regiones donde la escasez de agua es más latente, destacaba las oportunidades de disponer de fuentes alternativas de agua a la par de la disponibilidad de infraestructuras para reutilizar agua (Mesa-Pérez y Berbel, 2020). Sin embargo, en ambos casos se le da relevancia a convencer a la sociedad de la seguridad de regar productos agrícolas con agua regenerada.

Analizando ahora en profundidad los resultados de Andalucía, región marco en el caso de España, consideramos importante destacar por qué el agua regenerada puede jugar un rol de fuente alternativa al agua superficial y subterránea en Andalucía. La mayor parte de la población de Andalucía se encuentra en el litoral, lo que supone que el agua tratada por las estaciones depuradoras vaya directamente al mar sin poder darle un segundo uso como si recibe el agua depurada vertida en río, que es reutilizada indirectamente 
río abajo. Es por ello por lo que la regeneración puede proveer unos flujos hídricos constantes en las zonas costeras. Sin embargo, la situación de partida en Andalucía es preocupante, existe un 13\% de la población cuya agua no es tratada. Pese a ello, en Andalucía se tratan aproximadamente $700.000 \mathrm{hm}^{3}$ al año, de los cuales apenas $41.000 \mathrm{hm}^{3}$ se reutilizan, un 5,93\%. Otras zonas del mediterráneo como Murcia o la Comunidad Valenciana reutilizan el $72 \%$ y $48 \%$ respectivamente. Además, del agua reutilizada en Andalucía, apenas el 2,5\% va destinado a agricultura, siendo su principal destino el riego de jardines y campos de golf $(70 \%)$. Sin embargo, la cantidad de agua necesaria en un año normal, de acuerdo con la información de la Junta de Andalucía, para riego agrícola es de 3.912 millones de $\mathrm{hm}^{3}$. La reutilización podría ayudar a paliar las necesidades hídricas del campo andaluz.

El análisis DAFO realizado a los agentes claves en Andalucía nos muestra tres principales preocupaciones: escasez de agua, percepción de la sociedad y la legislación existente. La escasez de agua provocada por la sequía y el mal estado de las aguas subterráneas hacen necesario el estudiar posibles fuentes hídricas alternativas, teniendo ventaja la reutilización sobre la desalación por su coste inferior (El coste del agua regenerada es de $0.4 € / \mathrm{m} 3$, mientras el del agua desalada 0.6-0.8€/m3; [Cabrera et al. (2019)]. En cuanto a la percepción de la sociedad, aunque el Gobierno está actualizando la regulación al respecto de reutilización, aún contamos con un marco normativo obsoleto que no está adaptado a los requerimientos del Reglamento Europeo 2020/741. Sin embargo, ésta es también la principal oportunidad identificada por los actores claves y expertos cuestionados por SuWaNu-Europe, que destacaron que el marco normativo futuro solucionará los problemas existentes hasta el momento en relación con estándares de calidad. El nuevo marco regulatorio deberá ayudar también a concienciar sobre los beneficios de la reutilización y la recuperación de costes a la sociedad, circunstancia tan necesaria para consumidores y distribuidores de la cadena alimenticia. Finalmente, la última oportunidad encontrada en el proyecto es la disponibilidad de tecnología y casos de éxitos previos que demuestran que la regeneración es una fuente segura no solo para agricultura, sino también para consumo humano, como se puede ver en los casos de éxito de Chipre o Israel.

\section{Conclusiones}

El proyecto SuWaNu-Europe a través de la caracterización de las regiones de los países integrantes del proyecto y del análisis DAFO de los agentes clave permite proveer una imagen de la situación en Europa y Andalucía al respecto de la reutilización. Estos resultados son especialmente útiles ahora que el nuevo Reglamento Europeo presenta un marco legislativo proactivo para la reutilización y cuya aplicación será de obligado cumplimiento en junio de 2023. Sin embargo, una legislación homogénea a nivel europeo no podrá por sí sola fomentar la reutilización, ya que las oportunidades y barreras no son homogéneas en cada región de Europa. Es por ello por lo que su implementación deberá estar adaptada a cada región y/o país europeo. Además, las barreras que se han identificado son complejas, están interrelacionadas y afectan a diferentes actores (agricultores, autoridades, técnicos, consumidores, etc.) de los que se exige una cooperación necesaria para que la nueva legislación tenga el efecto deseado pero que tienen una percepción diferente del uso de aguas regeneradas y el problema de la escasez de agua. Por tanto, el diálogo entre los agentes implicados y las estrategias que pongan en común las diferentes visiones serán clave para extender el uso de aguas regeneradas en aquellas regiones como Andalucía donde este recurso aún no está suficientemente explotado.

Los resultados aquí expuestos demuestran que aquellos países con mayor disponibilidad de agua están más preocupados en la falta de infraestructuras y el alto coste de implementar la reutilización, mientras que los países del sur, que ya cuentan con amplias infraestructuras de regadío, lo ven como una oportunidad. Lo que sí queda claro es que, al igual que lo destacaron investigaciones previas (Mainali et al., 2011), concienciar a la sociedad de las virtudes y, sobre todo, de la seguridad del agua regenerada es clave para poder fomentar con éxito el riego agrícola con agua regenerada.

\section{Referencias}

Barua, A. (2013). Methods for Decision-Making in Survey Questionnaires Based on Likert Scale. Journal of Asian Scientific Research, 3(1), 35-38.

Berbel, J., \& Esteban, E. (2019). Droughts as a catalyst for water policy change. Analysis of Spain, Australia (MDB), and California. Global Environmental Change, 58(July), 101969. https://doi.org/10.1016/j.gloenvcha.2019.101969

Cabrera Marcet, E., Gomez Selles, E., Soriano Olivares, J., \& Teso-March, D. (2019). Eco-Layouts in Water Distribution Systems. Journal of Water Resources Planning and Management, 145(1), 111.

Croasmun, J. T., \& Ostrom, L. (2011). Using Likert-Type Scales in the Social Sciences. Journal of Adult Education, 40(1), 19-22. https://doi.org/10.1007/s10640-011-9463-0 
European Commission. Directive 2000/60/EC of the European Parliament and of the Council establishing a framework for Community action in the field of water policy (2000).

European Commission. (2019). The European Green Deal. https://doi.org/10.1017/CBO9781107415324.004

European Parliament. (2020). Regulation (EU) 2020/741 of 25 May 2020 on minimum requirements for water reuse. Official Journal of the European Union.

Hristov, J., Barreiro-Hurle, J., Salputra, G., Blanco, M., \& Witzke, P. (2021). Reuse of treated water in European agriculture: Potential to address water scarcity under climate change. Agricultural Water Management, 251(March), 106872. https://doi.org/10.1016/j.agwat.2021.106872

Mainali, B., Ngo, H. H., Guo, W. S., Pham, T. T. N., Wang, X. C., \& Johnston, A. (2011). SWOT analysis to assist identification of the critical factors for the successful implementation of water reuse schemes. Desalination and Water Treatment, 32(1-3), 297-306. https://doi.org/10.5004/dwt.2011.2714

MARM. (2010). Plan Nacional de Reutilización.

Mesa-Pérez, E., \& Berbel, J. (2020). Analysis of barriers and opportunities for reclaimed wastewater use for agriculture in Europe. Water (Switzerland), 12(8), 12082308. https://doi.org/10.3390/w12082308

Mesa-Pérez, E., Expósito, A., Casielles, R., \& Berbel, J. (2020). SWOT Analysis of Reclaimed Water Use for Irrigation in Southern Spain. In Water Reuse Within a Circular Economy Context (Vol. 2). Various.

Morote, Á. F., Olcina, J., \& Hernández, M. (2019). The use of non-conventional water resources as a means of adaptation to drought and climate change in semi-arid regions: South-eastern Spain. Water (Switzerland), 11(1). https://doi.org/10.3390/w11010093

Shannon, M. A., Bohn, P. W., Elimelech, M., Georgiadis, J. G., Marĩas, B. J., \& Mayes, A. M. (2008) Science and technology for water purification in the coming decades. Nature, 452(7185), 301-310. https://doi.org/10.1038/nature06599

SuWaNu Europe Project. (2019a). Deliverable 1.1 Regional state of play analyses. Retrieved from https://suwanu-europe.eu/water-recycle-project-documents/

SuWaNu Europe Project. (2019b). Reclaimed Water for Agricultural Irrigation Systems. Retrieved May 10, 2020, from https://suwanu-europe.eu/

SuWaNu Europe Project. (2020). Deliverable 2.1 - Report on SWOT and PEST analyses for implementation of reuse practices. Retrieved from https://suwanu-europe.eu/water-recycle-projectdocuments/ 